\title{
Changes in Device-Measured Physical Activity Patterns in U.K. Adults Related to the First COVID-19 Lockdown
}

\author{
Andrew P. Kingsnorth and Mhairi Patience \\ Loughborough University
}

Dale W. Esliger and Nicola J. Paine

Loughborough University

\author{
Elena Moltchanova \\ University of Canterbury \\ Matthew Hobbs \\ University of Canterbury
}

\begin{abstract}
The response to COVID-19 resulted in behavioral restrictions to tackle the spread of infection. Initial data indicates that step counts were impacted by lockdown restrictions; however, there is little evidence regarding changes of light and moderate to vigorous physical activity (MVPA) behavioral intensities. In this study, participants were asked to provide longitudinal wearable data from Fitbit devices over a period of 30 weeks, from December 2019 to June 2020. Self-assessed key worker status was captured, along with wearable estimates of steps, light activity, and MVPA. Bayesian change point analyses of data from 97 individuals found that there was a sharp decrease of 1,473 steps (95\% credible interval [CI] [-2,218, -709]) and light activity minutes $(41.9 ; 95 \%$ CI $[-54.3,-29.3])$, but an increase in MVPA minutes $(11.7 ; 95 \%$ CI $[2.9,19.4])$ in the mean weekly totals for nonkey workers. For the key workers, the total number of steps (207; 95\% CI [-788, 1,456]) and MVPA minutes increased (20.5; 95\% CI [12.6, 28.3]) but light activity decreased by an average of $46.9 \mathrm{~min}$ (95\% CI [-61.2, -31.8]). Interestingly, the change in steps was commensurate with that observed during Christmas $(1,458 ; 95 \%$ CI $[-2,286,-554])$ for nonkey workers and behavioral changes occurred at different time points and rates depending on key worker status. Results indicate that there were clear behavioral modifications before and during the initial COVID-19 lockdown period, and future research should assess whether any behavioral modifications were sustained over time.
\end{abstract}

Keywords: behavior, digital health, Fitbit

As of the beginning of February 2021, severe acute respiratory syndrome coronavirus-2 had infected more than 104.5 million individuals and had caused more than 2.3 million deaths worldwide (Johns Hopkins University \& Medicine, 2020). Named coronavirus disease 2019 (COVID-19), the outbreak began in December 2019 (World Health Organization, 2020) and has changed the lives of individuals around the world due to the direct effects of the infectious disease and the transmission mitigation methods implemented by their governments. While the economic cost such as a $5.2 \%$ contraction of global gross domestic product in 2020 is starting to be estimated (The World Bank, 2020), many of the other and longer-term effects of COVID-19 remain unknown. The U.K. government enforced behavioral "lockdown" restrictions on the March 23, 2020 which required individuals to stay at home and only to leave for limited purposes including shopping, medical

Kingsnorth and Patience shared joint first authorship. Kingsnorth, Patience, Esliger, and Paine are with the School of Sport, Exercise and Health Sciences, Loughborough University, Loughborough, Leicestershire, United Kingdom; and the National Centre for Sport and Exercise Medicine, Loughborough University, Loughborough, Leicestershire, United Kingdom. Moltchanova is with the School of Mathematics and Statistics, University of Canterbury, Christchurch, Canterbury, New Zealand. Paine is also with the Leicester Biomedical Research Centre-Lifestyle, Loughborough, Leicestershire, United Kingdom. Hobbs is with the School of Health Sciences, College of Education, Health and Human Development, University of Canterbury, Christchurch, Canterbury, New Zealand; and the GeoHealth Laboratory, Geospatial Research Institute, University of Canterbury, Christchurch, Canterbury, New Zealand. Kingsnorth (a.kingsnorth@lboro.ac.uk) is corresponding author. needs, travel for work (when they could not work from home), and to practice social distancing, hand washing, and self-isolation (GOV.UK, 2020). An additional restriction was to limit exercise to $1 \mathrm{hr}$ of outdoor exercise per day (GOV.UK, 2020), which has the potential to both positively and negatively impact on the daily routines of the population. Enforcing behavioral restrictions could influence the capability, motivation, and opportunity to conduct a behavior (Michie, van Stralen, \& West, 2011), and considering over a third of adults in England do not meet the current physical activity (PA) guidelines (Sport England, 2020a), any modifications in behavior, could have important implications for population health immediately after the pandemic and beyond.

There is emerging evidence that the nationwide lockdowns resulted in both a reduction of PA behaviors (Sánchez-Sánchez et al., 2020), and increases in PA, but also increases in sitting time (Romero-Blanco et al., 2020). U.K. data obtained by the Office for National Statistics indicates that compared with time use from 2014/2015, adults on average spent four more minutes keeping fit during the pandemic (Office for National Statistics, 2020). In addition, a study commissioned by Sport England revealed a mixed picture with $41 \%$ of adults reporting less activity and $31 \%$ more activity during the weekend of 3rd to 6th April (Sport England, 2020b). While providing interesting insights into physical behaviors during the pandemic on a relatively large set of respondents, this evidence is limited by both recall and self-report bias issues, and differences in questioning methods utilized.

Accelerometers are now commonly used to determine population level PA due to their feasibility in large-scale epidemiological 
studies (Doherty et al., 2017; Hamer et al., 2020; Lee \& Shiroma, 2014). But devices require deployment to quantify baseline activity levels and research processes are not always agile enough to react to natural experiments such as the changes in behaviors associated with events such as COVID-19. However, citizen science or the public involvement in scientific research is growing (Irwin, 2020), and 18\% of adults within the United Kingdom reported having wearable technology including smart watches or fitness trackers (Ofcom, 2020). Despite there being concerns over the ability of wearable devices to measure population level PA (Troiano, Stamatakis, \& Bull, 2020), the rapid onset of the COVID-19 outbreak precluded the deployment of research grade accelerometers. Therefore, harnessing the data of individuals that already own wearable devices and have been historically collecting data could provide a unique opportunity to understand PA behavioral patterns before, during, and after the COVID-19 pandemic. Indeed, researchers have begun to utilize longitudinal wearable device data in the form of step counts to indicate that PA levels decreased during countrywide lockdowns (Tison et al., 2020), which could also be related to the strictness of the lockdown measures introduced (Pépin et al., 2020), or occupational adaptations. Evidence from wearable manufacturer Fitbit early in the pandemic, highlighting European countries exhibiting a 7-38\% decline in step counts during the third week of March, would appear to further support this (Fitbit, 2020).

While the evidence relating to step count provides a device assessed measure of PA volume, commercial wearable devices such as Fitbits can partition movement behaviors into intensities categories of sedentary, light, moderate, and vigorous PA (Van Blarigan et al., 2017). These categories can be equated to cut points utilized by research grade accelerometers and exploring changes in PA intensity could therefore identify contextual changes in behavior that may represent adaptations to activities of daily living. For example, it is plausible that the enforced lockdown may have eroded incidental PA related to commuting to and from work, which is often at a light intensity; however, liberated time may have been reallocated to higher intensity PA (e.g., exercise), due to the planned and structured nature of the activities (Caspersen, Powell, \& Christenson, 1985). Utilizing data from individuals that have collected data before and during the COVID-19 lockdown could provide an interesting opportunity to study behavior in the absence of research grade accelerometers. Therefore, the aims of this study were to (a) investigate the influence of the initial COVID-19 lockdown period on PA behaviors by utilizing data from wearable Fitbit devices and (b) if any changes differed by demographic classifications.

\section{Methods}

\section{Study Design}

Data were obtained from individuals who had worn a Fitbit device before and during the first lockdown period. Participants were eligible to take part if they were an adult ( $\geq 18$ years of age), a U.K. citizen, owned and used a Fitbit device from December 2019 and were able to ambulate without walking aids, and had no functional/ mobility problems. The length of Fitbit ownership was used to determine a relative baseline for all participants and was confirmed by receiving data downloads for December. The study was approved by the Loughborough University Human Participants Ethical Sub-Committee (1419).

Data were requested from participants from April to July 2020; however, this related to PA conducted from December 2019 to June 2020. Participants were recruited through a convenience sample via series of posts on the social media platforms Twitter, Facebook, and Instagram including targeted posts to Fitbit user groups on Facebook. Interested participants were directed to an online survey platform (Online Surveys; Jisc, Bristol, UK) to initially confirm eligibility and then provide informed consent. After the questionnaire was completed, PA behavior data were then collected from the participants.

\section{Measurements}

Questionnaire. All eligible participants who provided informed consent completed an online questionnaire that included questions on demographics (age, postcode, height and weight, and socioeconomic status questions) and health and well-being (current health status, mental health, and social support). As part of the demographic questions, self-reported key worker status was confirmed via the question "Are you currently classed as a key worker during the Coronavirus pandemic?" to investigate occupational-related differences in behavior due to the continuation of working outside the home during the pandemic lockdown period. After the questionnaire was completed, a researcher then contacted the participant using contact details provided to request PA data access.

The questionnaire was launched in April 2020 and reviewed in June for uptake and feasibility. The initial mechanism of wearable PA data collection was for participants to be contacted by the research team via email. To increase the efficiency of the process, it was decided to allow participants to submit their wearable data via email immediately if they wished to. Sufficient text and video guidance were provided within the questionnaire to allow participants to do this independently. The option for the study team to contact participants was also still provided.

Wearable data. The Fitbit (Fitbit, San Francisco, CA) device was chosen due to the availability of a user export function which can produce daily summaries for up to a months' worth of data. To be eligible for this study, participants were required to have worn their Fitbit devices from December 2019 to provide sufficient lead time to the pandemic to act as a suitable baseline period. Any model of Fitbit device was available to be included within the study. Fitbit data can be exported via the individual's user account in up to 31 days at a time and the following PA metrics were available: calories burned, activity calories, steps, distance, floors, and minutes in sedentary, lightly active ( $<3$ metabolic equivalent of task [METs]), fairly active (3-5.9 METs), and very active intensities ( $\geq 6$ METs) (Van Blarigan et al., 2017). For this reason, categories data were provided as daily summaries, with one value for each of the variables per day.

\section{Data Processing}

Unlike research grade devices that have been extensively studied, the data processing algorithms of commercial devices are less well understood. After reviewing the data, the inconsistent nature of how the Fitbit algorithm works classified certain whole days $(1,440 \mathrm{~min}$ or $24 \mathrm{hr})$ as entirely sedentary. Due to this shortcoming with the variation in how sedentary time is categorized, it was not analyzed within this study. The remaining categories were therefore investigated and categorized as light (lightly active), moderate (fairly active), and vigorous (very active) PA (Van Blarigan et al., 2017). In addition, the combined category of moderate to vigorous physical activity (MVPA; $\geq 3$ METs) was formed by combining both fairly and very active variables to align with U.K. government guideline terminology (Department of Health and Social Care, 2019). 
As the data collection method utilized provided daily summaries of intensity variables, an assessment nonwear was required to ensure only representative days of data were included within the analyses. Unlike timestamped accelerometer data that often utilizes wear-time criteria of at least $10 \mathrm{hr}$ of wear over four or more valid days (Troiano et al., 2008), daily summaries do not provide the level of granularity to enact those criteria. A step-based threshold of $\geq 1,500$ steps/day has been previously used to determine valid days (Chu et al., 2017; Mikkelsen et al., 2020), which was derived within a pedometer study on both waist and wrist devices (TudorLocke, Barreira, \& Schuna, 2015). It was therefore decided that weekly totals were deemed valid if four or more valid days $(\geq 1,500$ steps/day) were provided.

\section{COVID-19 Contextual Information}

The daily summaries of participants were collapsed into Monday to Sunday weekly averages to aid in interpretation of the data, starting with Week 1 from December 2, 2019 to December 8, 2019. The United Kingdom went into a national (all four nation) lockdown on March 23, 2020, requiring that individuals stay at home, certain businesses close, and that social gathering cease. The easing of lockdown restrictions differed between the U.K. government and the other devolved governments.

\section{Statistical Analyses}

Step count, light, and MVPA variables were summarized into weekly averages and then visualized over time and stratified by sex, country of origin, and by key worker status. Rather than simply compare the activity levels before and after a fixed date, we have let the date of change (the change point) be determined by the model. Such an approach potentially takes into account the possibility that the change in behavior may not have happened instantaneously the moment the restrictions were imposed. The model was fitted within a Bayesian framework, as it is more flexible and particularly suitable for fitting a regression model with an unknown change point. The change point is thus treated as a parameter, and the model provides estimates and uncertainty bounds for it. For examples of similar applications please see Kürüm et al. (2017) and Elliott and Shope (2003).

Bayesian inference is based on posterior distributions of the parameters. The posterior distributions are obtained by combining the knowledge of the data-generating mechanism expressed via likelihood with the prior information on the parameters expressed via prior distributions. The posterior means form the parameter estimates, and the $95 \%$ central credible intervals (95\% CIs) are defined as the intervals which contain $95 \%$ of the posterior distribution mass.

Model comparison can be done by comparing model-specific deviance information criteria (DIC), which are similar in nature to the classical Akaike Information Criterion and are evaluated based on the combination of the model complexity and fit (Spiegelhalter, Best, Carlin, \& van der Linde, 2002). Better models have smaller DICs, and the difference of three is considered sufficient evidence for a statistically better model.

Within Bayesian framework, one can directly evaluate posterior probabilities of a particular statement being true, that is, $\operatorname{Pr}($ Statement|Evidence). Posterior probabilities are often called Bayesian $p$ values. Unlike classical frequentist $p$ values, the greater they are, the more support a particular statement has. The modeling details are as follows (key worker status stratification):
For the person ID in week $W$, we have assumed the response $Y_{\mathrm{ID}, W, K}$ to have a normal distribution with the expected activity $\mu$ postulated to have the following structure:

$$
\begin{aligned}
& \mu_{\mathrm{ID}, W, K}=a+b_{K} X_{W}+c_{K} I_{W>W_{K}^{*}} \\
& +d_{K} I_{W>W_{K}^{*}}\left(W-W_{K}^{*}\right)+\xi_{\mathrm{ID}}+\omega_{W},
\end{aligned}
$$

where $K$ is 1 for nonkey worker and 2 for key worker,

$X_{W}=1$ if the week $W$ falls within Christmas-New Year holiday (defined as the 2 weeks spanning December 23, 2019 to January 5, 2020, and 0 otherwise), and

$I_{W>W^{*}}=1$ if the week $W$ occurs after week $W^{*}$.

Here $W^{*}$ is the time points at which the lockdown effect is supposed to have taken place. In our model, it is a parameter, rather than a constant. That is, it is not fixed and it is allowed to be different for key workers and nonkey workers.

Finally, $\xi_{\text {ID }}$ is a participant-specific random effect, which takes into account the unbalanced repeated measurements design, and $\omega_{\mathrm{W}}$ is the week-specific residual accounting for possible temporal autocorrelation.

In the regression part of the model, $a$ refers to the average preCOVID step count, $b$ is the decrease in the average pre-COVID step count due to Christmas-New Year holiday, $c$ is the immediate decrease in average step count due to COVID, and $d$ is the linear trend in average count post-COVID lockdown.

For the number of steps per week, the log-linear model was found to be better than the linear one. For further modeling details please see the Supplementary Material (available online).

\section{Results}

\section{Descriptive Statistics}

Table 1 shows the study sample characteristics $(n=97)$. The mean age was 37 years and largely female (92\%), White British (96\%), have a high household income and mainly with an undergraduate degree or above $(65 \%)$. Fitbit compliance was high with on average 28 valid weeks of data and $89 \%$ (187/210) of valid days available to be included within the Fitbit data analyses. Results were investigated and stratified by gender, country of origin and by key worker status. There were clear differences for behavioral variables when the data were stratified by key worker status, and the aggregated means for steps, light activity, and MVPA categories, split by key worker status are outlined within Figure 1.

\section{Bayesian Inference}

Step count. Figure 2 shows posterior means and the respective $95 \%$ credible envelopes for the average number of weekly steps over time and by key and nonkey worker. The DICs for average steps per week are shown in Table S1 in Supplementary Material (available online).

The parameter estimates are shown in Table 2. In summary, for the nonkey workers, there is a clear change point $W_{1}^{*}$ on March 28 with 95\% CI 10-22 March. For the key workers, the change point $W_{2}^{*}$ is estimated to have occurred around March 29 with a much wider $95 \%$ CI (February 10 to May 11). This is also obvious in the much smoother curve. For nonkey workers, the initial sharp drop in steps was estimated to be 1,474 steps (95\% CI [709, 2,219]). This is comparable to the Christmas and New Year holiday. For the key workers there was, in fact a slight increase, on average, in activity: 
Table 1 Descriptive Statistics of the Study Sample

\begin{tabular}{|c|c|c|}
\hline Characteristics & Mean/n (\%) & $S D$ \\
\hline Age (years) & 37.35 & 12.76 \\
\hline Self-reported height $(\mathrm{cm})$ & 165.18 & 7.43 \\
\hline Self-reported weight $(\mathrm{kg})$ & 71.69 & 16.55 \\
\hline \multicolumn{3}{|l|}{ Sex } \\
\hline Female & $89(92)$ & \\
\hline Male & $8(8)$ & \\
\hline \multicolumn{3}{|l|}{ Ethnicity } \\
\hline White British, English, Northern Irish, Scottish, or Welsh & $93(96)$ & \\
\hline White Irish & $1(1)$ & \\
\hline White other & $2(2)$ & \\
\hline White and Black Caribbean & $1(1)$ & \\
\hline \multicolumn{3}{|l|}{ Household income } \\
\hline Below $£ 15 \mathrm{k}$ & $2(2)$ & \\
\hline$£ 15-£ 25 \mathrm{k}$ & $10(10)$ & \\
\hline$£ 25-£ 40 \mathrm{k}$ & $24(25)$ & \\
\hline$£ 40-£ 60 \mathrm{~K}$ & $34(35)$ & \\
\hline Above $£ 60 \mathrm{~K}$ & $27(28)$ & \\
\hline \multicolumn{3}{|l|}{ Highest qualification } \\
\hline Postgraduate degree & $18(19)$ & \\
\hline An undergraduate, first or foundation degree & $48(50)$ & \\
\hline Professional qualification & $13(13)$ & \\
\hline School degrees & $14(14)$ & \\
\hline N/A & $4(4)$ & \\
\hline \multicolumn{3}{|l|}{ Country of origin } \\
\hline England & $62(64)$ & \\
\hline Northern Ireland & $1(1)$ & \\
\hline Scotland & $32(32)$ & \\
\hline Wales & $2(2)$ & \\
\hline \multicolumn{3}{|l|}{ Key workers } \\
\hline Yes & $33(34)$ & \\
\hline No & $64(66)$ & \\
\hline \multicolumn{3}{|l|}{ Fitbit analyses } \\
\hline Average number of valid weeks per participant & 28.12 & 4.13 \\
\hline Average number of valid days per participant & 187.33 & 34.30 \\
\hline
\end{tabular}

206 steps $(95 \%$ CI $[-788,1,456])$. Both groups then showed gradual increase in steps. For the key workers, there was a strong positive increase of an average $1.7 \%$ per week (95\% CI [0.5\%, $3.4 \%]), \operatorname{Pr}\left(d_{1}>0\right)=0.96$. For the nonkey workers, the increase was much smaller $0.9 \%$ and the $95 \%$ CI $[-0.2 \%, 1.8 \%]$ included 0 . Interestingly, as shown in Figure 3 the posterior distributions for $b$ and $c_{2}$ are almost identical, meaning that the effects of lockdown on step count for nonkey workers are exactly comparable to that of the holiday period at Christmas and New year.

Light PA. The DICs for light activity are shown in Table S2 in Supplementary Material (available online). The maximal model was the best one for light activity as it was clearly statistically better than the model without change points $(\triangle \mathrm{DIC}=18)$ as well as the one without distinction by area $(\triangle \mathrm{DIC}=2.8)$. The resulting dynamics are shown below in Figure 3, and the estimated coefficients are reported in Table 3. There were considerable differences in the effect of the Christmas/New Year holiday on the light activity levels. While the key workers had considerable decreases (an average of $34 \mathrm{~min} /$ week), the nonkey workers experienced an average decrease of only $4 \mathrm{~min}$, not statistically different from zero. The change points were very sharply defined demonstrated by the narrower credible intervals for $W^{*}$. There was a clear sharp decrease in the amount of light activity for all groups with the averages ranging between 40 and $46 \mathrm{~min} /$ week and all credible intervals lying below zero. This initial decrease is generally more pronounced than the Christmas holiday. $\operatorname{Pr}\left(c_{K}>b_{K}\right)=$ 0.875 and 0.999 for the key and nonkey workers, respectively.

Moderate to vigorous physical activity. The DICs for MVPA are shown in Table S3 in Supplementary Material (available online) and the parameter estimates for the best model are shown in Table 4. Figure 4 shows a definite increase in minutes after the lockdown for both nonkey and key workers, $\operatorname{Pr}\left(c_{1}>0\right)>0.999$ and $\operatorname{Pr}\left(c_{2}>0\right)=0.989$, respectively. The increase was very 


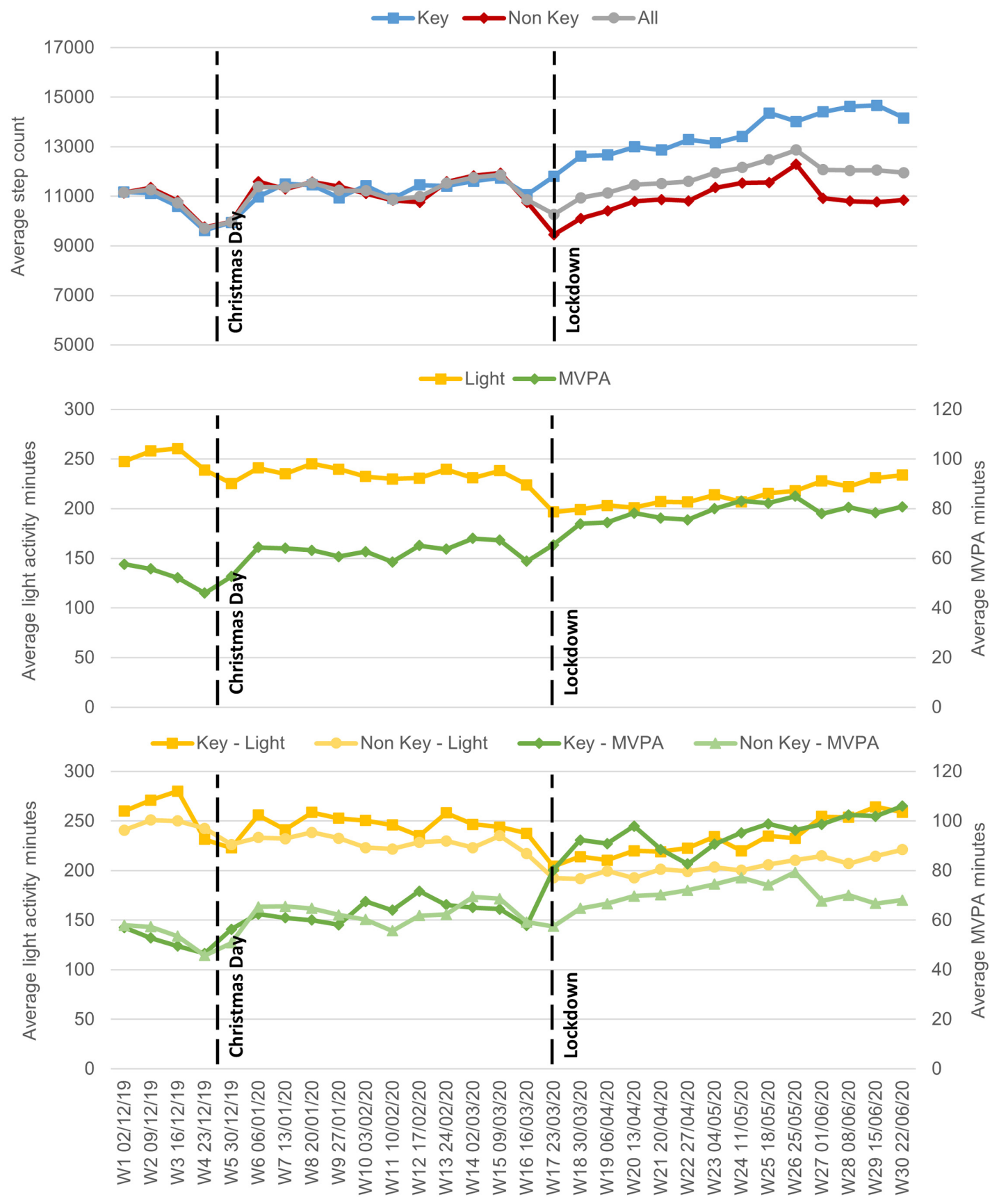

Figure 1 - Average steps, light activity, and MVPA minutes over 30 weeks of Fitbit monitoring. MVPA= moderate to vigorous PA; Light = light activity; PA = physical activity.

sharp for the key workers as is demonstrated by the very narrow $95 \%$ CI for $W_{1}^{*}$. For the key workers, the trend continues, $\left.\operatorname{Pr}\left(d_{2}>0\right)=0.997\right)$. For the nonkey workers, the $95 \% \mathrm{CI}$ for the trend $d_{1}$ includes 0 and $\operatorname{Pr}\left(d_{1}>0\right)=0.235$. In fact, a slight downward turn of an average 30 s/week has been estimated.

\section{Discussion}

This study provides evidence as to the extent to which PA behaviors were influenced by the initial COVID-19 lockdown period by utilizing data from Fitbit wearable devices collected from U.K. citizens. Findings in this study show changes in PA behavior as defined by steps, light activity, and MVPA minutes per week before, during, and following the U.K. lockdown. While light PA sharply decreased in response to the lockdown period, MVPA minutes increased. Results also show that changes in behavior varied between individuals classified as key and nonkey workers. For example, whereas light activity declined prior to the introduction of the COVID-19 lockdown for both groups (-46.9 and $-41.9 \mathrm{~min})$, Bayesian change-point analyses showed that MVPA increased at different time points and rates between key workers (20.5 min; March 19) than nonkey workers (11.7 min; April 9). 


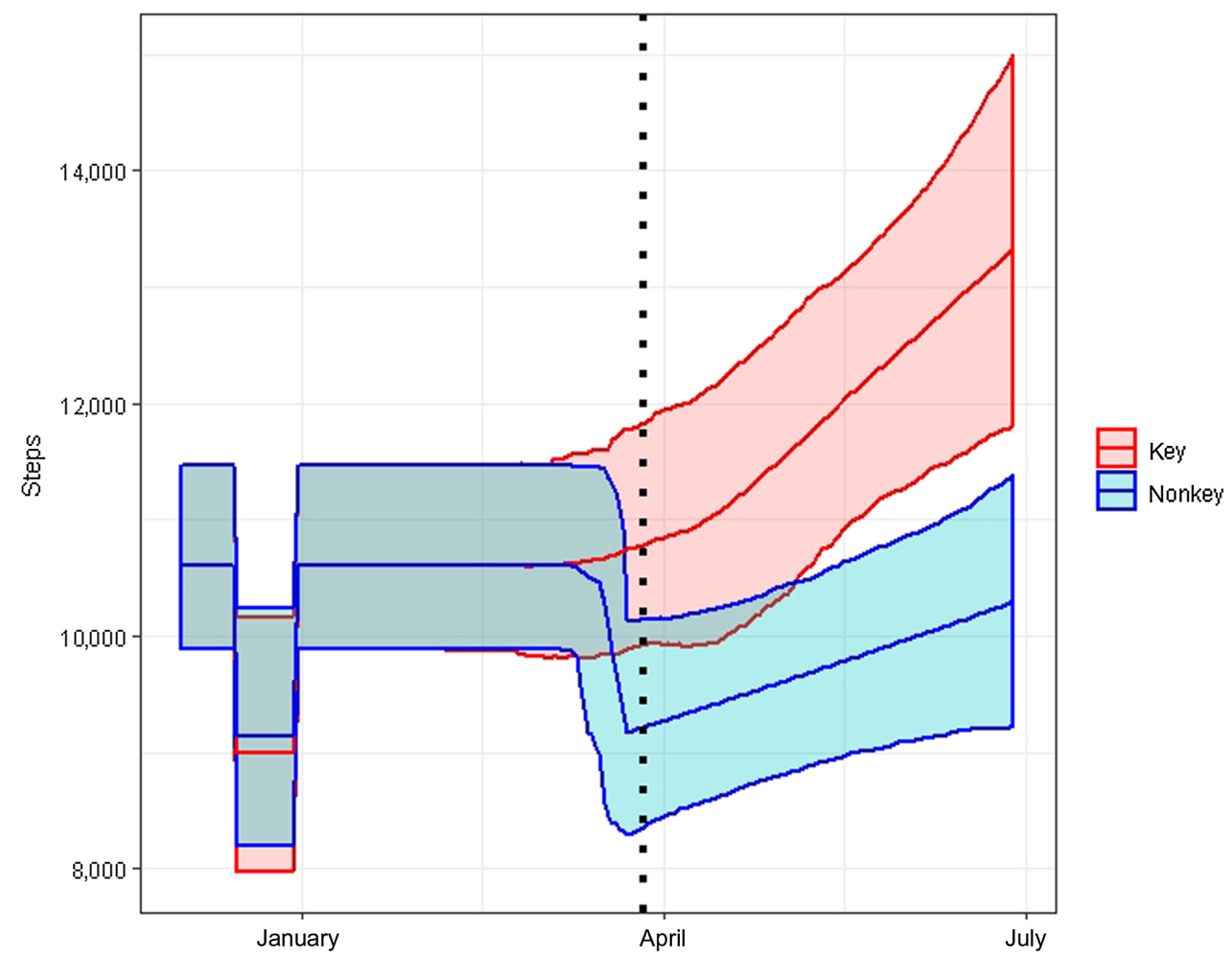

Figure 2 - The observed (solid lines) and fitted posterior (dotted lines) means and $95 \%$ credible envelopes for the average number of steps for the key and nonkey workers. The thick dotted line indicates the beginning of the official lockdown. (Note. Predictions are model averaged over the posterior distribution of change points. Therefore, the transitions are smooth.)

Table 2 Fitted Coefficients for the Model of the Average Number of Steps (Regression Coefficients Have Been Exponentiated for Easier, Percentage, and Interpretation)

\begin{tabular}{lcc}
\hline Parameter & Posterior mean & Posterior 95\% credible interval \\
\hline Pre-COVID $a$ & 10,618 & {$[9,887,11,464]$} \\
Christmas-New Year change $b_{1}$ (key) & $-1,609$ & {$[-2,527,-599]$} \\
Christmas-New Year change $b_{2}$ (nonkey) & $-1,458$ & {$[-2,286,-554]$} \\
Immediate change $c_{1}($ key) & 207 & {$[-788,1,456]$} \\
Immediate change $c_{2}$ (nonkey) & $-1,473$ & {$[-2,218,-709]$} \\
Postchange recovery $d_{1}$ (key) & $1.7 \%$ & {$[0.5 \%, 4.4 \%]$} \\
Postchange recovery $d_{2}$ (nonkey) & $0.9 \%$ & {$[-0.2 \%, 1.8 \%]$} \\
Change point $W_{1}^{*}$ (key) & March 29 & {$[$ February 12, May 11$]$} \\
Change point $W_{2}^{*}$ (nonkey) & March 18 & {$[$ March 10, March 22] } \\
\hline
\end{tabular}

According to a recent Sport England publication, 37\% of the U.K. population do not meet the current PA guidelines and of those $25 \%$ conduct less than 30 min of activity a week (Sport England, 2020a). As such, promoting adequate levels of PA is already a major public health issue (World Health Organization, 2013), and previous microsimulation evidence has suggested that a $10 \%$ reduction in PA would be associated with increased weight gain (An, 2020), and that existing low levels of PA may have been compounded by the COVID-19 pandemic (Dunton, Do, \& Wang, 2020). In contrast, recommendations suggest that individuals and organizations should consider using PA as a strategy to maintain physical and mental health during pandemics such as COVID-19, which could also have the potential to reduce the severity of infections (Jurak et al., 2020; Sallis \& Pratt, 2020). Despite these commentaries and simulations, empirical evidence confirming such changes in PA behavior are lacking.

In this study, changes were seen for step count which began to decrease even before a lockdown was implemented. This corroborates an analysis of step count data from multiple countries that perhaps indicates a preemptive response of individuals to concerns around the impact and prevalence of COVID-19 within the community, or perhaps due to initial less stringent restrictions observed (Pépin et al., 2020). For nonkey workers, the initial decrease in steps was more pronounced compared with key workers. For context, this initial sharp drop in step count for 


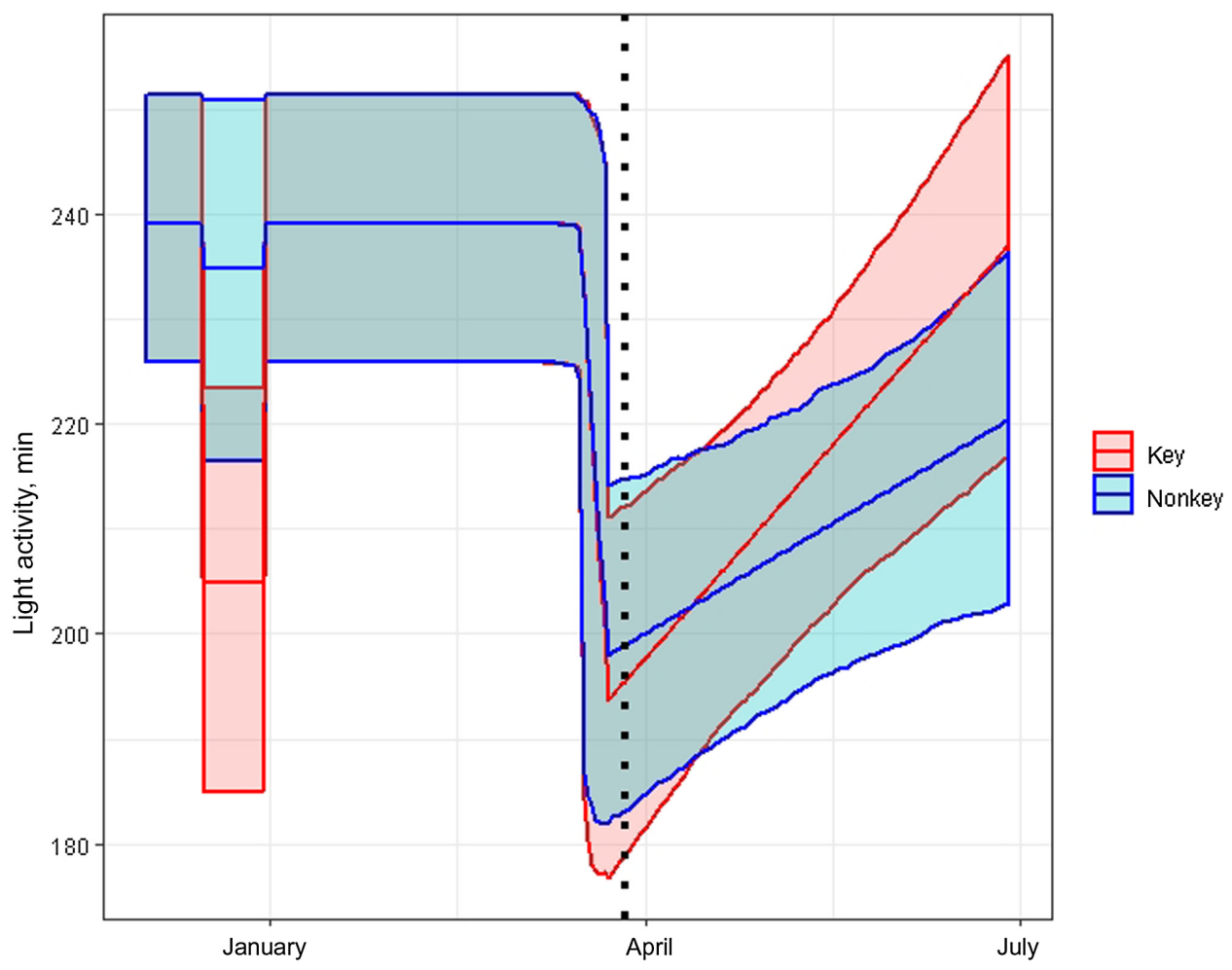

Figure 3 - The fitted posterior means and 95\% credible envelopes for the average minutes of light activity for the key and nonkey workers. The thick dotted line indicates the beginning of the official lockdown. (Note. Predictions are model averaged over the posterior distribution of change-points; therefore, the transitions are smooth.)

Table 3 Fitted Coefficients for the Model of Light Activity (Minutes per Week)

\begin{tabular}{lcc}
\hline Parameter & Posterior mean & Posterior 95\% credible interval \\
\hline Pre-COVID $a$ & 239.1 & {$[225.9,251.3]$} \\
Christmas-New Year change $b_{1}$ (key) & -34.3 & {$[-49.6,-18.5]$} \\
Christmas-New Year change $b_{2}$ (nonkey) & -4.2 & {$[-17.5,8.6]$} \\
Immediate change $c_{1}$ (key) & -46.9 & {$[-61.2,-31.8]$} \\
Immediate change $c_{2}$ (nonkey) & -41.9 & {$[-54.3,-29.3]$} \\
Postchange recovery $d_{1}$ (key) & 3.1 & {$[1.4,4.8]$} \\
Postchange recovery $d_{2}$ (nonkey) & 1.6 & {$[0.2,2.9]$} \\
Change point $W_{1}^{*}$ (key) & March 19 & {$[$ March 16, March 22] } \\
Change point $W_{2}^{*}$ (nonkey) & March 19 & {$[$ March 16, March 22] } \\
\hline
\end{tabular}

nonkey workers was almost identical to the decrease seen over the Christmas and New Year holiday period. In contrast, for key workers, the Bayesian break-point analysis confirmed a slight increase in step count, and over time, step count also increased the most for key workers while the step count increase over time for nonkey workers was much smaller. While only in children, emerging U.S. evidence has showed that they performed less PA and engaged in more sedentary behavior during the earlyCOVID-19 period as compared with before the pandemic (Dunton et al., 2020). In addition, while children used remote/streaming services for activity classes and lessons during the early-COVID-19 period (Dunton et al., 2020), little is known for adult populations regarding the switching of behaviors that could be representative of adaptations to home isolation or working, or the increased opportunity and motivation to be more PA (Michie et al., 2011). Nevertheless, step count does not account of the intensity of PA. Therefore, it is important to ascertain whether there have been any intensity related shifts in behavior due to the imposed restrictions.

In this study, the combined fairly active and very active category of PA (MVPA) increases in the immediate weeks after lockdown starts. A confirmed effect was observed via the break-point analyses which highlighted increased MVPA after lockdown for all individuals, regardless of key worker status. Nevertheless, this effect was only persistent for key workers. These results could indicate a shift away from activities of daily living to more purposeful PA, which could be initiated from the restrictions in place upon one exercise per day which was allowed during the lockdown. However, this effect seemed to stabilize over time into June but remained higher than 
Table 4 Fitted Coefficients for the Model of MVPA (Minutes per Week)

\begin{tabular}{lcc}
\hline Parameter & Posterior mean & Posterior 95\% credible interval \\
\hline Pre-COVID $a$ & 60.4 & {$[53.1,67.5]$} \\
Christmas-New Year change $b_{1}$ (key) & -12.1 & {$[-21.5,-2.1]$} \\
Christmas-New Year change $b_{2}$ (nonkey) & -14.0 & {$[-21.3,-6.1]$} \\
Immediate change $c_{1}$ (key) & 20.5 & {$[12.6,28.3]$} \\
Immediate change $c_{2}$ (nonkey) & 11.7 & {$[2.9,19.4]$} \\
Postchange recovery $d_{1}$ (key) & 1.3 & {$[0.4,2.1]$} \\
Postchange recovery $d_{2}$ (nonkey) & -0.5 & {$[-2.0,0.7]$} \\
Change point $W_{1}^{*}$ (key) & March 19 & {$[$ March 16, March 22] } \\
Change point $W_{2}^{*}$ (nonkey) & April 9 & [February 26, April 27] \\
\hline
\end{tabular}

Note. $\mathrm{MVPA}=$ moderate to vigorous PA; PA= physical activity.

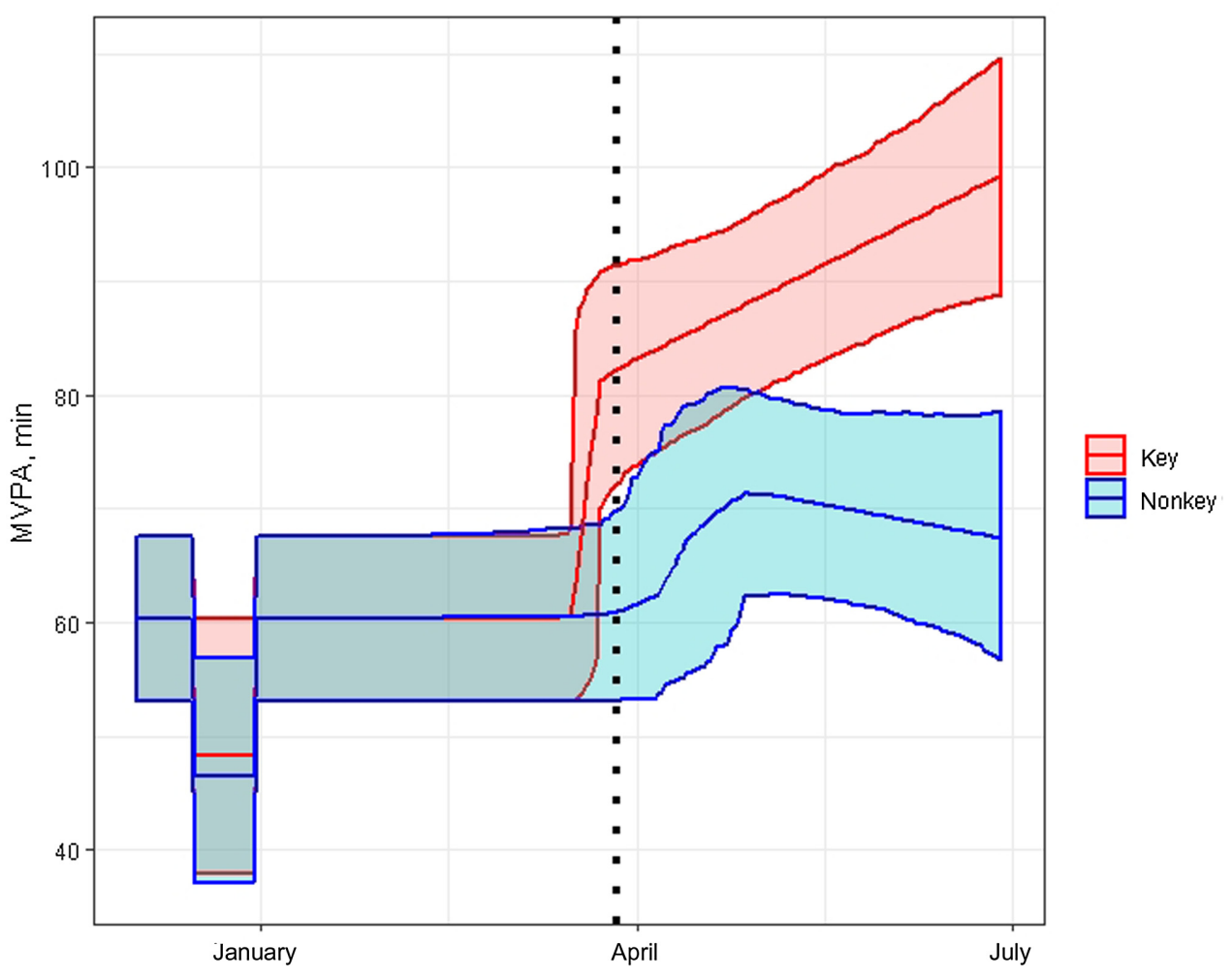

Figure 4 - The fitted posterior means and 95\% credible envelopes for the average minutes of MVPA for the key and nonkey workers. The thick dotted line indicates the beginning of the official lockdown. (Note. Predictions are model averaged over the posterior distribution of change points; therefore, the transitions are smooth). MVPA = moderate to vigorous PA; PA = physical activity.

prelockdown levels, particularly for key workers. It is widely acknowledged that regular PA is beneficial for both mental and physical health, and maintaining PA levels during the COVID-19 pandemic is vital to bolster the immune response and reduce the effect of negative risk factors such as age and obesity (Ainsworth \& $\mathrm{Li}, 2020$; Nieman, 2020). While this study provides some of the first empirical evidence of PA intensity behaviors during the pandemic, a follow-up is required to establish the impact of easing restrictions upon behavioral estimates and whether the above baseline changes of behavior stabilized or persisted over a longer period of time.

The current study has utilized a citizen science approach to overcome the limitation of a lack of baseline data as many wearable users have been collecting data on themselves before, during, and will continue to after the COVID-19 pandemic. Nevertheless, the sample was predominantly female, White, well educated and of a high household income. This notion is not new and is often well described within the literature (Paré, Leaver, \& Bourget, 2018), but does limit the generalizability of the current findings. The Fitbit device was chosen due to being a well-known and utilized brand that targets more general population use, in addition to the relative ease of use of exporting the variables from the dashboard and to eliminate between manufacturer errors, from their various algorithms. The proprietary nature of common commercial data interfaces with the user prohibit easy data harmonization that could provide additional insights from other sectors of society that may choose different wearable monitors. With Apple, Xiaomi, and 
Samsung reported to comprise of $31.1 \%, 11.8 \%$, and $9.4 \%$, respectively (IDC, 2020), of the market share, a cross platform analysis of data would therefore be an interesting proposal to really provide detailed insights of PA levels during the pandemic.

\section{Limitations}

There are some limitations within the data and study design that should be considered. Despite being longitudinal in nature and separating behavioral intensities from wearable data, the small homogenous sample does limit generalizability of the data. The geographic spread of the participants did capture a large proportion of the United Kingdom; yet the sample comprised mainly women of a White ethnicity. Further investigations with a larger group of more diverse, representative participants is required to confirm the findings presented within this study. This could be combined with a longitudinal analysis and data-sharing partnership with commercial companies to establish how stable the behavioral changes are, considering the partial opening up of society into the winter months. In addition, participants were sought who owned a Fitbit device prior to the pandemic. Those who routinely own and wear a wearable tracker may have been more motivated to be physically active, and therefore the impact of the initial lockdown could have differed from those who didn't wear a device.

Moreover, the validity of wearable devices such as Fitbits to quantify PA intensities has been called into question (Redenius, Kim, \& Byun, 2019), but not their ability to measure steps (Fuller et al., 2020). Utilizing the public to provide wearable device data can overcome the lack of agility to respond to rapid developing research situations. Nevertheless, the findings of the present study must be evaluated against the limitations of nonresearch grade monitors that can vary both within and between brands of wearable devices.

\section{Conclusions}

In conclusion, this study found evidence of behavioral changes to PA and step counts during the COVID-19 lockdown period, and specifically, these changes seem to be most pronounced in key workers. The increased opportunity and motivation through a reduction in other social activities and limit to one opportunity per day highlight possible beneficial effects of lockdown restrictions. However, future research should ascertain whether any increases in behavior were sustained over time. While this study utilized a citizen science approach to respond to the lack of a baseline period, partnerships with commercial wearable companies should be explored to replicate these methods in a more diverse population.

\section{Acknowledgments}

The authors would like to thank the participants for taking part in this study and for agreeing to provide their data during such a challenging time. The authors also thank VV for the useful discussions regarding their device usage and PA patterns while developing the project and the initial COVID19 lockdown period.

\section{References}

Ainsworth, B.E., \& Li, F. (2020). Physical activity during the coronavirus disease-2019 global pandemic. Journal of Sport and Health Science,
9(4), 291-292. PubMed ID: 32525096 doi:10.1016/j.jshs.2020. 06.004

An, R. (2020). Projecting the impact of the coronavirus disease-2019 pandemic on childhood obesity in the United States: A microsimulation model. Journal of Sport and Health Science, 9(4), 302-312. PubMed ID: 32454174 doi:10.1016/j.jshs.2020.05.006

Caspersen, C.J., Powell, K.E., \& Christenson, G.M. (1985). Physical activity, exercise, and physical fitness: Definitions and distinctions for health-related research. Public Health Reports, 100(2), 126-131. doi: $10.2307 / 20056429$

Chu, A.H.Y., Ng, S.H.X., Paknezhad, M., Gauterin, A., Koh, D., Brown, M.S., \& Müller-Riemenschneider, F. (2017). Comparison of wristworn Fitbit Flex and waist-worn ActiGraph for measuring steps in free-living adults. PLoS One, 12(2), e0172535. PubMed ID: 28234953 doi:10.1371/journal.pone.0172535

Department of Health and Social Care. (2019). UK Chief Medical Officers' physical activity guidelines. Retrieved from https://www.gov.uk/ government/publications/physical-activity-guidelines-uk-chiefmedical-officers-report

Doherty, A.s, Jackson, D., Hammerla, N., Plötz, T., Olivier, P., Granat, M.H., .. Wareham, N.J. (2017). Large scale population assessment of physical activity using wrist worn accelerometers: The UK biobank study. PLoS One, 12(2), e0169649. PubMed ID: 28146576 doi:10. 1371/journal.pone.0169649

Dunton, G.F., Do, B., \& Wang, S.D. (2020). Early effects of the COVID19 pandemic on physical activity and sedentary behavior in children living in the U.S. BMC Public Health, 20(1), 1351. PubMed ID: 32887592 doi:10.1186/s12889-020-09429-3

Elliott, M.R., \& Shope, J.T. (2003). Use of a Bayesian changepoint model to estimate effects of a graduated driver's licensing program. Journal of Data Science, 1(1), 43-63.

Fitbit. (2020). The impact of coronavirus on global activity. Retrieved from https://perma.cc/2JZ6-H3R2

Fuller, D., Colwell, E., Low, J., Orychock, K., Tobin, M.A., Simango, B., ... Taylor, N.G.A. (2020). Reliability and validity of commercially available wearable devices for measuring steps, energy expenditure, and heart rate: Systematic review. JMIR MHealth and UHealth, 8(9), e18694. PubMed ID: 32897239 doi:10.2196/ 18694

GOV.UK. (2020). Prime Minister's statement on coronavirus (COVID19). Retrieved from https://www.gov.uk/government/speeches/pmaddress-to-the-nation-on-coronavirus-23-march-2020

Hamer, M., Stamatakis, E., Chastin, S., Pearson, N., Brown, M., Gilbert, E., \& Sullivan, A. (2020). Feasibility of measuring sedentary time using data from a thigh-worn accelerometer. American Journal of Epidemiology, 189(9), 963-971. PubMed ID: 32219368 doi:10.1093/ aje/kwaa047

IDC. (2020). Wearable devices market share. Retrieved from https://www. idc.com/promo/wearablevendor/vendor

Irwin, A. (2020). No PhDs needed: How citizen science is transforming research. Retrieved from https://www.nature.com/articles/d41586018-07106-5

Johns Hopkins University \& Medicine. (2020). Coronavirus resource center. Retrieved from https://perma.cc/BQ4H-TX3N

Jurak, G., Morrison, S.A., Leskošek, B., Kovač, M., Hadžić, V., Vodičar, J., ... Starc, G. (2020). Physical activity recommendations during the coronavirus disease-2019 virus outbreak. Journal of Sport and Health Science, 9(4), 325-327. PubMed ID: 32426171 doi:10.1016/j. jshs.2020.05.003

Kürüm, E., Warren, J.L., Schuck-Paim, C., Lustig, R., Lewnard, J.A., Fuentes, R., ... Weinberger, D.M. (2017). Bayesian model averaging with change points to assess the impact of vaccination and public 
health interventions. Epidemiology, 28(6), 889-897. PubMed ID: 28767518 doi:10.1097/EDE.0000000000000719

Lee, I.-M., \& Shiroma, E.J. (2014). Using accelerometers to measure physical activity in large-scale epidemiological studies: Issues and challenges. British Journal of Sports Medicine, 48(3), 197-201. PubMed ID: 24297837 doi:10.1136/bjsports-2013-093154

Lunn, D.J., Thomas, A., Best, N., \& Spiegelhalter, D. (2000). WinBUGS -A Bayesian modelling framework: Concepts, structure, and extensibility. Statistics and Computing, 10(4), 325-337. doi:10.1023/A: 1008929526011

Michie, S., van Stralen, M.M., \& West, R. (2011). The behaviour change wheel: A new method for characterising and designing behaviour change interventions. Implementation Science, 6(1), 42. doi:10.1186/ 1748-5908-6-42

Mikkelsen, M.-L.K., Berg-Beckhoff, G., Frederiksen, P., Horgan, G., O’Driscoll, R., Palmeira, A.L., ... Larsen, S.C. (2020). Estimating physical activity and sedentary behaviour in a free-living environment: A comparative study between Fitbit Charge 2 and Actigraph GT3X. PLoS One, 15(6), e0234426. PubMed ID: 32525912 doi:10. 1371/journal.pone.0234426

Nieman, D.C. (2020). Coronavirus disease-2019: A tocsin to our aging, unfit, corpulent, and immunodeficient society. Journal of Sport and Health Science, 9(4), 293-301. PubMed ID: 32389882 doi:10.1016/j. jshs.2020.05.001

Ofcom. (2020). Media literacy tracker 2019. Retrieved from https://www. ofcom.org.uk/_data/assets/pdf_file/0028/196372/adults-media-useand-attitudes-2020-data-tables.pdf

Office for National Statistics. (2020). Coronavirus and how people spent their time under lockdown: 28 March to 26 April 2020. Retrieved from https://perma.cc/GW6V-65DD

Paré, G., Leaver, C., \& Bourget, C. (2018). Diffusion of the digital health self-tracking movement in Canada: Results of a national survey. Journal of Medical Internet Research, 20(5), e177. PubMed ID: 29720359 doi:10.2196/jmir.9388

Pépin, J.L., Bruno, R.M., Yang, R.-Y., Vercamer, V., Jouhaud, P., Escourrou, P., \& Boutouyrie, P. (2020). Wearable activity trackers for monitoring adherence to home confinement during the COVID-19 pandemic worldwide: Data aggregation and analysis. Journal of Medical Internet Research, 22(6), e19787. PubMed ID: 32501803 doi:10.2196/19787

R Core Team. (2013). R: A language and environment for statistical computing. $R$ Foundation for Statistical Computing. Retrieved from http://www.r-project.org/

Redenius, N., Kim, Y., \& Byun, W. (2019). Concurrent validity of the Fitbit for assessing sedentary behavior and moderate-to-vigorous physical activity. BMC Medical Research Methodology, 19(1), 29. PubMed ID: 30732582 doi:10.1186/s12874-019-0668-1

Romero-Blanco, C., Rodríguez-Almagro, J., Onieva-Zafra, M.D., ParraFernández, M.L., Prado-Laguna, M., \& Hernández-Martínez, A. (2020). Physical activity and sedentary lifestyle in university students: Changes during confinement due to the COVID-19 pandemic. International Journal of Environmental Research and Public Health, 17(18), 6567. doi:10.3390/ijerph17186567
Sallis, J.F., \& Pratt, M. (2020). Multiple benefits of physical activity during the Coronavirus pandemic. Revista Brasileira de Atividade Física \& Saúde, 25, 1-5. doi:10.12820/rbafs.25e0112

Sánchez-Sánchez, E., Ramírez-Vargas, G., Avellaneda-López, Y., Orellana-Pecino, J.I., García-Marín, E., \& Díaz-Jimenez, J. (2020). Eating habits and physical activity of the spanish population during the COVID-19 pandemic period. Nutrients, 12(9), 2826. doi:10.3390/ nu12092826

Spiegelhalter, D.J., Best, N.G., Carlin, B.P., \& van der Linde, A. (2002). Bayesian measures of model complexity and fit. Journal of the Royal Statistical Society: Series B (Statistical Methodology), 64(4), 583639. doi:10.1111/1467-9868.00353

Sport England. (2020a). Active lives adult survey November 2018/19 Report. Retrieved from https://sportengland-production-files.s3.euwest-2.amazonaws.com/s3fs-public/2020-04/Active\%20Lives\%20 Adult\%20November\%2018-19\%20Report..pdf?BhkAy2K28pd9b DEz_NuisH12ppuqJtpZ

Sport England. (2020b). Coronavirus - Research into how the coronavirus crisis has affected people's activity levels and attitudes towards exercise. Retrieved from https://perma.cc/KE3S-KQKH

The World Bank. (2020). The global economic outlook during the COVID-19 pandemic: A changed world. Retrieved from https:// www.worldbank.org/en/news/feature/2020/06/08/the-global-economicoutlook-during-the-covid-19-pandemic-a-changed-world

Tison, G.H., Avram, R., Kuhar, P., Abreau, S., Marcus, G.M., Pletcher, M.J., \& Olgin, J.E. (2020). Worldwide effect of COVID-19 on physical activity: A descriptive study. Annals of Internal Medicine, 173(9), 767-770. doi:10.7326/m20-2665

Troiano, R.P., Berrigan, D., Dodd, K.W., Mâsse, L.C., Tilert, T., \& McDowell, M. (2008). Physical activity in the United States measured by accelerometer. Medicine \& Science in Sports \& Exercise, 40(1), 181-188. PubMed ID: 18091006 doi:10.1249/mss.0b013e $31815 \mathrm{a} 51 \mathrm{~b} 3$

Troiano, R.P., Stamatakis, E., \& Bull, F.C. (2020). How can global physical activity surveillance adapt to evolving physical activity guidelines? Needs, challenges and future directions. British Journal of Sports Medicine, 54(24), 1468-1473. PubMed ID: 33239352 doi:10.1136/bjsports-2020-102621

Tudor-Locke, C., Barreira, T.V., \& Schuna, J.M. (2015). Comparison of step outputs for waist and wrist accelerometer attachment sites. Medicine \& Science in Sports \& Exercise, 47(4), 839-842. PubMed ID: 25121517 doi:10.1249/MSS.0000000000000476

Van Blarigan, E.L., Kenfield, S.A., Tantum, L., Cadmus-Bertram, L.A., Carroll, P.R., \& Chan, J.M. (2017). The fitbit one physical activity tracker in men with prostate cancer: Validation study. JMIR Cancer, 3(1), e5. PubMed ID: 28420602 doi:10.2196/ cancer.6935

World Health Organization. (2013). Global action plan for the prevention and control of NCDs 2013-2020. Retrieved from https://apps.who.int/ iris/bitstream/handle/10665/94384/9789241506236_eng.pdf;jsession id=084532D7C4D54D47AB689B68A076D51A? sequence=1

World Health Organization. (2020). Q\&A on coronaviruses (COVID-19). Retrieved from https://perma.cc/8NRC-5B26 